\title{
Effects of selective cyclooxygenase-2 and nonselective cyclooxygenase inhibition on ischemic myocardium
}

\author{
Michael P. Robich, MD, MSPH, ${ }^{\mathrm{a}, \mathrm{b}}$ Louis M. Chu, MD, ${ }^{\mathrm{a}}$ Jun Feng, MD, PhD, ${ }^{\mathrm{d}}$ Thomas A. Burgess, MA, ${ }^{\mathrm{d}}$ \\ Roger J. Laham, MD, ${ }^{\mathrm{c}}$ Cesario Bianchi, MD, PhD, ${ }^{\mathrm{d}}$ and Frank W. Sellke, MD ${ }^{\mathrm{a}, \mathrm{b}, \mathrm{d}}$
}

Objective: We explored effects of nonselective cyclooxygenase and selective cyclooxygenase 2 inhibition on collateral development in a model of chronic myocardial ischemia. We hypothesized that cyclooxygenase 2 inhibitors would negatively effect angiogenic and inflammatory pathways.

\begin{abstract}
Methods: Yorkshire swine were made chronically ischemic by placing an ameroid constrictor on the left circumflex coronary artery. Swine were divided into 3 groups and given no drug (control, $n=7$ ), a nonselective cyclooxygenase inhibitor (naproxen $400 \mathrm{mg}$ daily, $\mathrm{n}=7$ ), or a selective cyclooxygenase 2 inhibitor (celecoxib $200 \mathrm{mg}$ daily, $\mathrm{n}=7$ ). After 7 weeks, coronary angiography was performed. Myocardial function and microvascular reactivity were assessed. Serum and myocardial tissue were analyzed for prostaglandin levels and markers of inflammation and angiogenesis.
\end{abstract}

Results: The celecoxib group demonstrated significantly increased mean arterial pressure and decreased left ventricular function. Myocardial perfusion in the celecoxib group was similar to control value but less than in the naproxen group. Coronary microvascular contraction in the collateral-dependent territory was increased in the naproxen group but minimally affected in the celecoxib group. Oxidative stress and apoptosis were increased in the celecoxib group. Expression of angiogenic markers vascular endothelial growth factor and phospho-endothelial nitric oxide synthase (ser1177) and tissue levels of prostacyclin were decreased in both celecoxib and naproxen groups. The naproxen group had diminished endostatin expression.

Conclusions: Selective and nonselective cyclooxygenase inhibition are more complex in effect than previously published, but they did not decrease collateral-dependent blood flow to the myocardium in our model of chronic myocardial ischemia. (J Thorac Cardiovasc Surg 2010;140:1143-52)

Selective inhibitors of the inducible isoform of cyclooxygenase (COX-2) were developed to provide relief of pain associated with arthritis, surgery, and other conditions while lowering the incidence of gastrointestinal complications relative to nonselective nonsteroidal anti-inflammatory drugs (NSAIDs). ${ }^{1}$ Selective COX-2 inhibitors have been

\footnotetext{
From the Department of Surgery, ${ }^{a}$ Division of Cardiothoracic Surgery, Warren Alpert School of Medicine, Brown University, Providence, RI; the Department of Surgery, Division of Cardiothoracic Surgery, ${ }^{\mathrm{b}}$ and the Department of Medicine, Division of Cardiology, ${ }^{\mathrm{c}}$ Beth Israel Deaconess Medical Center, Harvard Medical School, Boston, Mass; and the Department of Surgery, ${ }^{\mathrm{d}}$ Division of Cardiothoracic Surgery, Rhode Island Hospital, Providence, RI.

Supported by the National Heart, Lung and Blood Institute (F.S.W.) grants RO1HL46716, RO1HL69024, and RO1HL85647; by the National Institutes of Health (M.P.R.) grant 5T32-HL0074; and by the Irving Bard Memorial Fellowship (M.P.R., L.M.C.).

Disclosures: F.W.S. has research support from Ikaria (Clinton, NJ) and Orthologic (Tempe, Ariz) and is a consultant for Novo Nordisk (Princeton, NJ) and Cubist Pharmaceuticals (Lexington, Mass). F.W.S. is a consultant for the law firms representing Pfizer (Princeton, NJ) in the Bextra/Celebrex litigation, but no funding was received for this study, and there was no consultation or notification regarding this study.

Read at the 90th Annual Meeting of The American Association for Thoracic Surgery, Toronto, Ontario, Canada, May 1-5, 2010.

Received for publication April 22, 2010; revisions received May 28, 2010; accepted for publication June 28, 2010; available ahead of print Sept 1, 2010.

Address for reprints: Frank W. Sellke, MD, Division of Cardiothoracic Surgery, Cardiovascular Research Center, Alpert Medical School, Brown University, 593 Eddy St, APC 424, Providence, RI 02903 (E-mail: fsellke@lifespan.org).

$0022-5223 / \$ 36.00$

Copyright (C) 2010 by The American Association for Thoracic Surgery

doi:10.1016/j.jtcvs.2010.06.057
}

purported, however, to increase the risk of adverse cardiovascular events, such as myocardial infarction, and to increase mortality. One widely prescribed selective COX-2 inhibitor, rofecoxib, was removed from the market in 2004 because of an increased incidence of adverse cardiovascular events observed during the VIGOR trial, which examined the effects of rofecoxib on arthritic pain relief. ${ }^{2}$ On the other hand, nonselective NSAIDs have also been implicated in increased cardiovascular events. ${ }^{3,4}$ As a consequence, both selective and nonselective NSAID COX inhibitors carry a "black box warning" from the United States Food and Drug Administration related to the perceived risk of increased cardiovascular events.

COX is responsible for the production of thromboxane (TX) $\mathrm{A}_{2}$ in platelets, which facilitates thrombosis and is a vasoconstrictor in most vascular beds. $\mathrm{TXA}_{2}$ is antagonized by low-dose aspirin. On the other hand, in endothelium COX catalyzes the formation of prostacyclin $\left(\mathrm{PGI}_{2}\right)$, a substance known to be an antithrombotic and a vasodilator. In the so-called FitzGerald hypothesis, the key to potential cardiovascular side effects with selective COX-2 inhibitors was purported to be related to the balance between the prothrombotic and antithrombotic prostaglandins. In 2006, FitzGerald $^{5}$ published a mouse study in which selective inhibition by knockout of COX-2 or deletion of the $\mathrm{PGI}_{2}$ receptor yielded an increase in thrombotic events and elevated blood 


$$
\begin{aligned}
& \text { Abbreviations and Acronyms } \\
& \begin{aligned}
\mathrm{ADP} & =\text { adenosine diphosphate } \\
\mathrm{COX} & =\text { cyclooxygenase } \\
\mathrm{JNK} & =\text { c-Jun N-terminal kinase } \\
\mathrm{LCx} & =\text { left circumflex coronary artery } \\
\mathrm{NSAID}^{\prime} & =\text { nonsteroidal anti-inflammatory drug } \\
\mathrm{PGF}_{1 \alpha} & =\text { prostaglandin } \mathrm{F}_{1 \alpha} \\
\mathrm{PGI}^{2} & =\text { prostacyclin } \\
\mathrm{TX} & =\text { thromboxane }
\end{aligned}
\end{aligned}
$$

pressure. Inhibition of COX-1, which simulates the effects of aspirin, reversed these effects.

Another theory consistent with increased cardiovascular risk from long-term selective COX-2 inhibition was proposed by $\mathrm{Wu}$ and associates. ${ }^{6}$ In their study, inhibition of COX-2 in cell culture led to decreased angiogenesis. In addition, the formation of vascular structures in Matrigel (BD Biosciences, San Jose, Calif) was accelerated in COX-2-overexpressing cells but attenuated in cells transfected with COX-2 small inhibitory RNA. This finding suggests that selective COX-2 inhibitors may lead to decreased angiogenesis and coronary collateral formation. With fewer (or less well developed) protective collateral vessels, patients may suffer more devastating outcomes during ischemic cardiac events. Coronary collateral development has been associated with reduced risks of death and myocardial injury after coronary artery occlusion. ${ }^{7}$

At this time, no studies have examined the effects of nonselective COX or selective COX-2 inhibitors on angiogenesis or myocardial perfusion in any large animal model of chronic myocardial ischemia. We hypothesized that inhibition of COX-2 would alter the productions of $\mathrm{TXA}_{2}$ and $\mathrm{PGI}_{2}$, negatively effect coronary collateral formation and proangiogenic pathways, and lead to diminished collateraldependent myocardial perfusion.

\section{METHODS \\ Study Design}

Yorkshire miniswine (Parsons Research, Amherst, Mass) were divided into 3 groups. One group received celecoxib at $200 \mathrm{mg}$ orally per day $(\mathrm{n}=8)$, another received naproxen at $400 \mathrm{mg}$ orally per day $(\mathrm{n}=10)$, and the third received no drug (control, $\mathrm{n}=8$ ). All animals received drug treatment for 7 weeks, beginning at the time of ameroid placement. All animals underwent ameroid constrictor placement on the proximal left circumflex coronary artery (LCx). For all surgical procedures, anesthesia was induced with ketamine hydrochloride $(10 \mathrm{mg} / \mathrm{kg}$ intramuscularly) and thiopental sodium $(2.5 \%)$ and maintained with a gas mixture of oxygen at 1.5 to $2 \mathrm{~L} / \mathrm{min}$ and $3.0 \%$ isoflurane. The animals were intubated and mechanically ventilated at 16 breaths/min. During the first procedure, the pericardium was opened through a left minithoracotomy, and a titanium ameroid constrictor (1.75-mm internal diameter; Research Instruments SW, Escondido, Calif) was placed around the proximal LCx. Seven weeks after ameroid placement, each swine was again anesthetized, the heart was exposed, and physiologic measurements were taken, followed by humane killing.
The swine were not given any medication to prevent or treat potential arrhythmias.

Once the heart had been removed, myocardial samples were rapidly frozen in liquid nitrogen for molecular studies, placed in $4^{\circ} \mathrm{C}$ Krebs solution for microvessel reactivity studies, or placed in $10 \%$ formalin for immunohistochemical studies.

All experiments were approved by the Beth Israel Deaconess Medical Center institutional animal care and use committee. Animals were cared for in compliance with the Harvard Medical Area institutional animal care and use committee and in accordance with the Principles of Laboratory Animal Care formulated by the National Society for Medical Research and the Guide for the Care and Use of Laboratory Animals (www.nap.edu/catalog/ 5140.html).

\section{Measurement of Global and Regional Myocardial Function}

Indices of global and regional left ventricular function were measured before animals were killed and included the following: systolic blood pressure, developed left ventricular pressure, positive and negative first derivatives of left ventricular pressure, and longitudinal and horizontal segmental shortening in the area at risk for 10 sequential beats, as determined with a Sonometrics system (Sonometrics Corp, London, Ontario, Canada).

\section{X-Ray Coronary Angiography}

$\mathrm{X}$-ray coronary angiography with iohexol (Omnipaque; GE Healthcare, Princeton, NJ) was carried out to ensure occlusion of the LCx and to assess collateral formation. Recorded images were interpreted by an interventional cardiologist (R.J.L.) blinded to the treatment groups. Angiographic collateral formation was assessed according to the blush score and Rentrop grading system, depending on the presence and extension of the collateral filling of coronary epicardial vessels.

\section{Microvessel Studies}

After cardiac removal, coronary arterioles ( $80-180 \mu \mathrm{m}$ in diameter) from the ischemic territory were dissected from the surrounding tissue and placed in an isolated microvessel chamber as described previously. ${ }^{8}$ After precontraction of microvessels by $30 \%$ to $50 \%$ of the baseline diameter with the $\mathrm{TXA}_{2}$ analog $\mathrm{U} 46619(0.1-1.0 \mu \mathrm{mol} / \mathrm{L})$, the microvascular responses to vasorelaxing agents sodium nitroprusside $\left(10^{-9}\right.$ to $10^{-4} \mathrm{~mol} / \mathrm{L}$, an endothelium-independent cyclic guanosine monophosphate-mediated vasodilator) and adenosine diphosphate (ADP, $10^{-9}-10^{-4} \mathrm{~mol} / \mathrm{L}$, an endothelium-dependent vasodilator) were evaluated. Responses to vasoconstricting agents endothelin $1\left(10^{-12}-10^{-7}\right)$ and serotonin $\left(10^{-9}-10^{-5}\right)$ were assessed in vessels that had not been precontracted. All drugs were applied extraluminally. Responses were defined as percentage relaxation of the preconstricted diameter for ADP and sodium nitroprusside and as percentage constriction of baseline diameter for endothelin 1 and serotonin. All reagents were obtained from Sigma-Aldrich (St Louis, Mo).

\section{Myocardial Perfusion Analysis}

Myocardial perfusion was determined with isotope-labeled microspheres $15 \mu \mathrm{m}$ in diameter (BioPAL Worcester, Mass) according to previously reported methods. ${ }^{9}$ Briefly, $1.5 \times 10^{7}$ gold-labeled microspheres were injected during temporary LCx occlusion at the time of ameroid placement to identify the area at risk. Lutetium (rest conditions) and europium (ventricular pacing at 150 beats $/ \mathrm{min}$ ) isotopically labeled microspheres were injected before the animal was killed. After the animal was killed, left ventricular sections were collected for isotope-labeled microsphere assay. The samples were exposed to neutron beams, and microsphere densities were measured with a gamma counter.

\section{Immunoblotting Studies}

Tissue lysate was made from the ischemic myocardium. A $60-\mu \mathrm{g}$ sample of total protein was fractionated by $4 \%$ to $20 \%$ gradient sodium dodecyl 
sulfate polyacrylamide gel electrophoresis (Invitrogen Corporation, Carlsbad, Calif) and transferred to polyvinylidene fluoride membranes (Millipore, Billerica, Mass). Each membrane was incubated with specific primary antibodies. Antibodies were obtained from Cell Signaling (Cell Signaling Technology, Inc, Danvers, Mass), BD Biosciences, and Abcam (Abcam Inc, Cambridge, Mass). Immune complexes were visualized with the enhanced chemiluminescence detection system (Amersham, Piscataway, NJ). Bands were quantified by densitometric evaluation of autoradiograph films. Ponceau staining was used to ensure equal protein loading.

\section{Immunofluorescence Double Staining for Dividing and Total Endothelial Cells}

Frozen sections (12 $\mu \mathrm{m}$ in thickness) of myocardium from the ischemic territories were formalin fixed. Antibodies against proliferation marker Ki67 (Abcam) and PECAM-1 (CD-31; R\&D Systems, Inc, Minneapolis, Minn) were simultaneously applied to the sections and incubated. Detection was obtained with appropriate secondary antibodies (Jackson ImmunoResearch Laboratories, Inc, West Grove, Pa). Sections were mounted in Vectashield with 4',6-diamidino-2-phenylindole (Vector Laboratories, Inc, Burlingame, Calif). Photomicrographs were taken with a Zeiss Axiolab microscope (Carl Zeiss, Inc, Thornwood, NY) equipped with a digital camera at 200× magnification (PhotoDoc-It; UVP, LLC, Upland, Calif). Total and dividing endothelial cells were counted in a blinded fashion. Data are presented as numbers of Ki-67-positive endothelial cells per square millimeter.

\section{Tissue TX and Tissue and Serum PGI $\mathrm{PL}_{2}$ Levels}

Tissue levels of stable TX and $\mathrm{PGI}_{2}$ breakdown products, 11-dehydro$\mathrm{TXB}_{2}$ and 6-keto prostaglandin $\mathrm{F}_{1 \alpha}\left(\mathrm{PGF}_{1 \alpha}\right)$, respectively, were measured by enzyme-linked immunosorbent assay (Neogen Corp, Lexington, Ky). Tissue lysates from the ischemic myocardium underwent liquid-liquid exchange, extraction, and concentration according to the manufacturer's recommendations. The samples were put into an antibody-coated plate along with horseradish peroxidase-conjugated 6-keto $\mathrm{PGF}_{1 \alpha}$. The plate was washed, and horseradish peroxidase substrate was added. The plate was incubated and then read at $650 \mathrm{~nm}$, and sample results were plotted against the standard curve.

Serum levels of 6-keto $\mathrm{PGF}_{1 \alpha}$ were measured by enzyme-linked immunosorbent assay (Neogen). Serum samples underwent liquid-liquid exchange, extraction, concentration, and assay as described previously.

\section{Myocardial Protein Oxidative Stress}

Dinitrophenylhydrazine-derivatized myocardial tissue homogenates were separated by $10 \%$ polyacrylamide gel electrophoresis and transferred to polyvinylidene fluoride membranes (Chemicon International, Inc, Temecula, Calif). The membranes were incubated with primary antibody specific to dinitrophenylhydrazine, followed by incubation with a horseradish peroxidase-linked secondary antibody. Immune complexes were visualized with the enhanced chemiluminescence detection system (Amersham).

\section{Myocardial Lipid Oxidative Stress}

Measurement of lipid peroxidation in the ischemic myocardium was carried out with a commercial kit to assess levels of free malondialdehyde, a reactive carbonyl compound produced during decomposition of lipid peroxides (Oxis International Inc, Foster City, Calif). Homogenates were incubated with $\mathrm{N}$-methyl-2-phenylindole in acetonitrile, probucol, and concentrated hydrochloric acid. Samples were centrifuged, and the malondialdehydecontaining supernatant was removed. Absorbance was measured at $586 \mathrm{~nm}$, and values were analyzed against a standard curve. Levels of malondialdehyde were corrected for protein concentration differences between samples.

\section{Quantification of Apoptotic Cells}

Apoptotic cells in the myocardium were identified with the ApopTag detection kit according to manufacturer's specifications (Millipore). At least
$1 \mathrm{~cm}^{2}$ of tissue was analyzed from each animal (4 per group). The number of cardiomyocytes found to be positive by terminal deoxynucleotidyl transferase-mediated deoxyuridine triphosphate nick end labeling was expressed as positive cells per square centimeter.

\section{Data Analysis}

All results are expressed as mean \pm SEM. Microvessel responses are expressed as percentage relaxation of the preconstricted diameter or as contraction of the baseline diameter and were analyzed with 2-way repeated measures analysis of variance with a post hoc Bonferroni test, which was applied to interactions of treatment and dose. Western blots were analyzed after digitization (ScanJet 4c; Hewlett-Packard Company, Palo Alto, Calif) with NIH ImageJ 1.33 software (National Institutes of Health, Bethesda, $\mathrm{Md})$. For data analysis, levels of phosphorylated proteins were normalized to total expression levels. Comparisons among the 3 groups were analyzed by 1-way repeated measures analysis of variance with Newman-Keuls multiple comparison post hoc test by means of GraphPad Prism 4 (GraphPad Software, Inc., La Jolla, Calif).

\section{RESULTS}

\section{Animal Model}

During the course of this study, there were 5 deaths: 1 in the control group, 1 in the celecoxib group, and 3 in the naproxen group. The animals that died in the naproxen and celecoxib groups showed symptoms consistent with heart failure, tachypnea, and abdominal ascites. These animals died an average of $32 \pm 1.2$ days after ameroid placement. There were 7 animals included in each group for analysis after 2 replacement animals were added to the naproxen group.

\section{Functional Data}

Heart rate was significantly lower in the celecoxib group than in the control group (control $138 \pm 17$ beats/min, naproxen $108 \pm 17$ beats/min, and celecoxib $90 \pm 4$ beats/min, $P=.04)$. Both mean arterial pressure and left ventricular pressure were significantly higher in the celecoxib group. In addition, segmental shortening was significantly higher in the celecoxib group than in the control and naproxen groups. The positive first derivative of left ventricular pressure over time, a preload- and afterload-independent measure of global left ventricular function, was lower in the celecoxib group than in the naproxen and control groups (Figure 1, $A-D$ ).

\section{Collateral-Dependent Blood Flow in the Ischemic Territory}

There was a significant increase in absolute blood flow at rest in the naproxen group. In all groups, absolute blood flow was unaffected by ventricular pacing ( 150 beats/ minute; Figure 2, $A$ and $B$ ). Collateral-dependent myocardial blood flows were similar in the celecoxib and control groups at rest and with pacing. There were no differences in left anterior descending coronary arterial flow among the groups at rest (control $0.86 \pm 0.17 \mathrm{~mL} /[\mathrm{min} \cdot \mathrm{g}]$, naproxen $1.22 \pm 0.18 \mathrm{~mL} /[\mathrm{min} \cdot \mathrm{g}]$, and celecoxib $1.00 \pm 0.18 \mathrm{~mL} /[\mathrm{min} \cdot \mathrm{g}], P=.36)$ or during paced conditions (control $0.96 \pm 0.13 \mathrm{~mL} /[\mathrm{min} \cdot \mathrm{g}]$, naproxen 

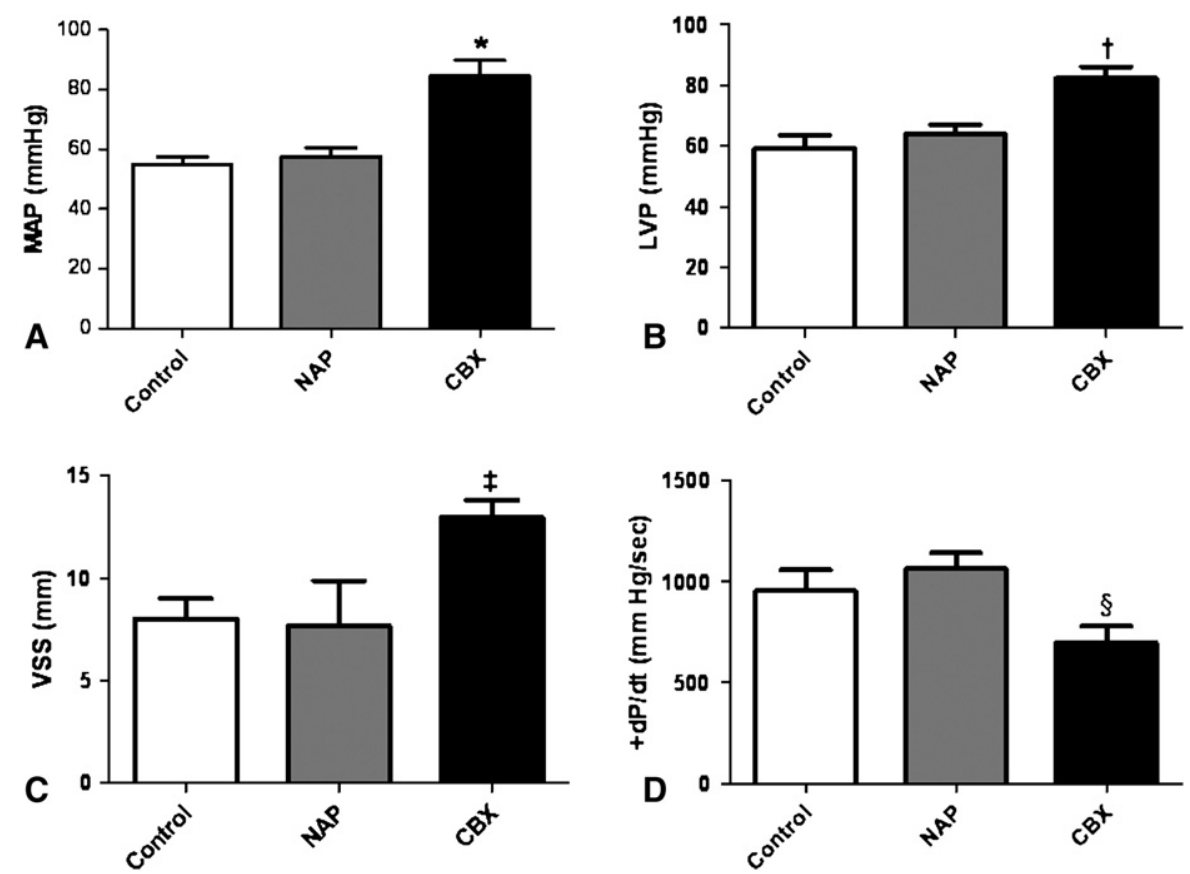

FIGURE 1. Myocardial functional data. A, Mean arterial blood pressure $(M A P)$ is increased in celecoxib (CBX) group (asterisk indicates $P<.001)$. B, Left ventricular pressure $(L V P)$ is increased in celecoxib group (dagger indicates $P=.004)$. C, Vertical segmental shortening $(V S S)$ is increased in celecoxib group (double dagger indicates $P=.02$ ). D, Positive first derivative of developed pressure over time $(+d P / d t)$ is decreased in celecoxib group (section mark indicates $P=.04)$. NAP, Naproxen.

$0.86 \pm 0.06 \mathrm{~mL} /[\mathrm{min} \cdot \mathrm{g}]$, and celecoxib $0.8600 \pm$ $0.18 \mathrm{~mL} /[\mathrm{min} \cdot \mathrm{g}], P=.83)$.

\section{Coronary Angiography}

In all cases, total LCx occlusion was observed during the final coronary angiogram, and there were no differences in observed Rentrop scores (control $0.89 \pm 0.3$, naproxen $1.4 \pm 0.38$, and celecoxib $1.6 \pm 0.37, P=.35)$. There were also no differences in observed blush scores among the groups (control $0.17 \pm 0.17$, naproxen $0.8 \pm 0.37$, and celecoxib $0.5 \pm 0.22, P=.25)$.

\section{Microvascular Function}

There were no differences in baseline microvessel diameter among groups (control $156 \pm 13 \mu \mathrm{m}$, naproxen $140 \pm 10 \mu \mathrm{m}$, and celecoxib $142 \pm 10 \mu \mathrm{m}, P=.60)$. Likewise, there were no differences among groups in the preconstricted diameter (control $96 \pm 5 \mu \mathrm{m}$, naproxen $86 \pm 13 \mu \mathrm{m}$, celecoxib $94 \pm$ $7 \mu \mathrm{m}, P=.78$ ) or percentage precontraction (control $-36.6 \% \pm 2 \%$, naproxen $-36.0 \% \pm 3.1 \%$, celecoxib $-34.1 \% \pm 1.4 \%, P=.73)$ of microvessels. There was a greater contraction response in the naproxen group to endothelin-1 than in the control and celecoxib groups. Serotonin-induced contraction was greater in the naproxen group than in both control and celecoxib groups. When examining the microvascular response to ADP, an endotheliumdependent vasorelaxing agent, the celecoxib group demonstrated decreased relaxation response at the highest dose of ADP relative to the control and naproxen groups. There were no differences among groups when the vessels were
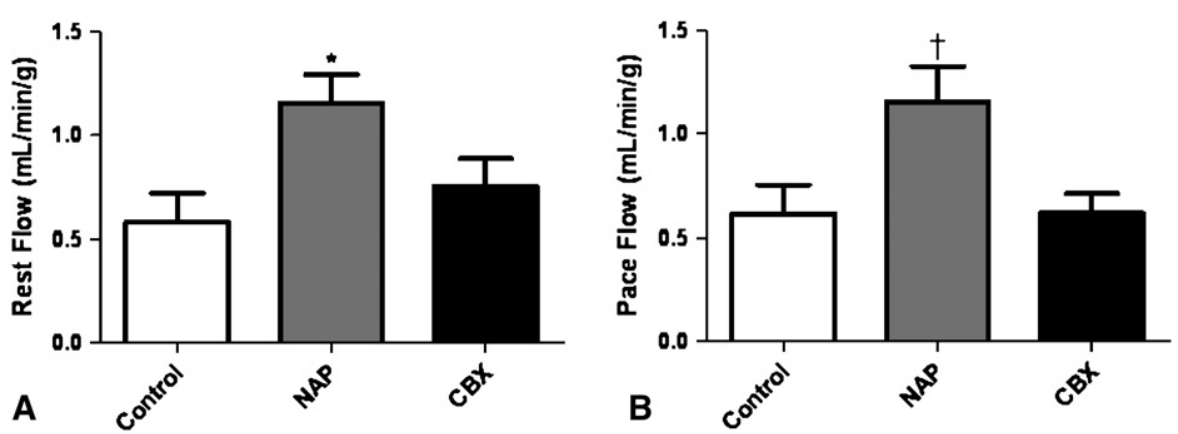

FIGURE 2. Blood flow in collateral-dependent territory. A, Blood flow to ischemic myocardium at rest is increased in naproxen (NAP) group (asterisk indicates $P=.03$. B, There is significantly increased blood flow to ischemic territory during ventricular pacing in naproxen group (dagger indicates $P=.02)$. 

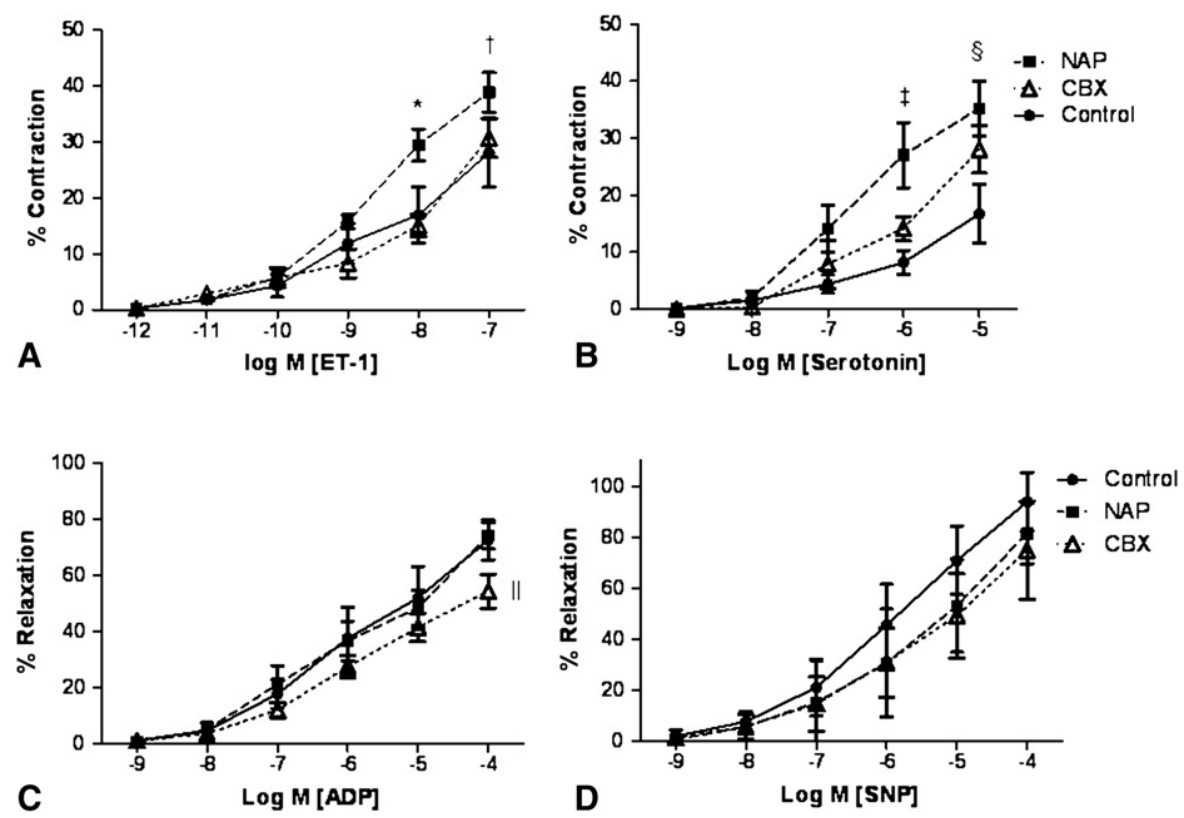

FIGURE 3. Microvascular responses. A, Microvessel responses to endothelin-1 (ET-1) demonstrate significantly increased contractions in naproxen (NAP) group at $10^{-8}$ (asterisk indicates $P=.001$ vs control and celecoxib $[C B X]$ ) and $10^{-9}$ (dagger indicates $P=.001$ vs control). Percentage contraction is not different between control and celecoxib groups at any concentration. B, Contraction responses to serotonin demonstrate increases in naproxen group at $10^{-6}$ (double dagger indicates $P=.001$ vs control and celecoxib) and $10^{-5}$ (section mark indicates $P=.04$ vs control). C, Relaxation responses to adenosine diphosphate $(A D P)$, endothelium-dependent vasorelaxation agent, show decreased microvascular responses in celecoxib group (parallel lines indicate $P=.01$ vs control and naproxen). D, Responses to sodium nitroprusside ( $S N P)$ are not different among groups $(P=.22)$.

subjected to sodium nitroprusside, an endotheliumindependent vasorelaxing agent (Figure 3, $A-D$ ).

\section{Levels of $\mathrm{PGI}_{2}$ and TX in the Ischemic Myocardium and Serum}

The naproxen group exhibited a marked decrease in tissue levels of $\mathrm{PGF}_{1 \alpha}$, whereas $\mathrm{PGF}_{1 \alpha}$ in the celecoxib group was slightly reduced relative to control. The naproxen and celecoxib groups both had markedly lower serum levels of $\mathrm{PGF}_{1 \alpha}$ than did the control group. Both the naproxen and celecoxib groups demonstrated a trend toward increased tissue levels of $\mathrm{TXB}_{2}$, although these differences did not reach statistical significance (Figure 4, $A-C$ ).

\section{Levels of Tissue Oxidant Stress}

The celecoxib group demonstrated significantly higher levels of protein oxidative stress in the ischemic territory of the left ventricle (Figure 5, A). Levels of tissue malondialdehyde (lipid oxidative stress) were similar among the groups (Figure 5, $B$ ).

\section{Quantification of Capillary Growth and Density}

Both the naproxen and celecoxib groups demonstrated fewer dividing endothelial cells than did the control group. In the assessment of the total number of endothelial cells in the ischemic myocardium, there were also significantly fewer cells in the naproxen and celecoxib groups than in the control group (Figure 6, $A-E$ ).

\section{Levels of Apoptosis}

The number of apoptotic cells in the ischemic myocardium in the celecoxib group was significantly increased relative to the control and naproxen groups (control $0.017 \pm 0.002$ cells $/ \mathrm{cm}^{2}$, naproxen $0.050 \pm 0.023$ cells/ $\mathrm{cm}^{2}$, celecoxib $0.092 \pm 0.047$ cells $\left./ \mathrm{cm}^{2}, P=.002\right)$.

\section{Immunoblotting}

In the examination of proangiogenic proteins, vascular endothelial growth factor and vascular endothelial growth factor receptor 2 expressions were decreased in the ischemic myocardium of both naproxen and celecoxib groups relative to the control group. Similarly, expressions of phospho-endothelial nitric oxide synthase (ser1177) were diminished in both naproxen and celecoxib groups. Expression of c-Jun Nterminal kinase (JNK) was increased in the celecoxib group relative to the control group. Expression of the antiangiogenic protein endostatin was significantly lower in the naproxen group than in the control and celecoxib groups (Figure 7, A-E). Expressions of phospho-Akt (ser473) were increased 1.4-fold in the naproxen and celecoxib groups, but this did not reach significance $(P=.10)$. There was also a trend toward increased tumor necrosis factor $\alpha$ expression in the celecoxib group relative to the control and naproxen groups $(P=.08)$. There were no differences among the groups in the myocardial expression levels of nuclear factor $\kappa \mathrm{B}(P=.11)$, extracellular signal-regulated kinase $(P=.76)$, or apoptosis-inducing factor $(P=0.55)$. 

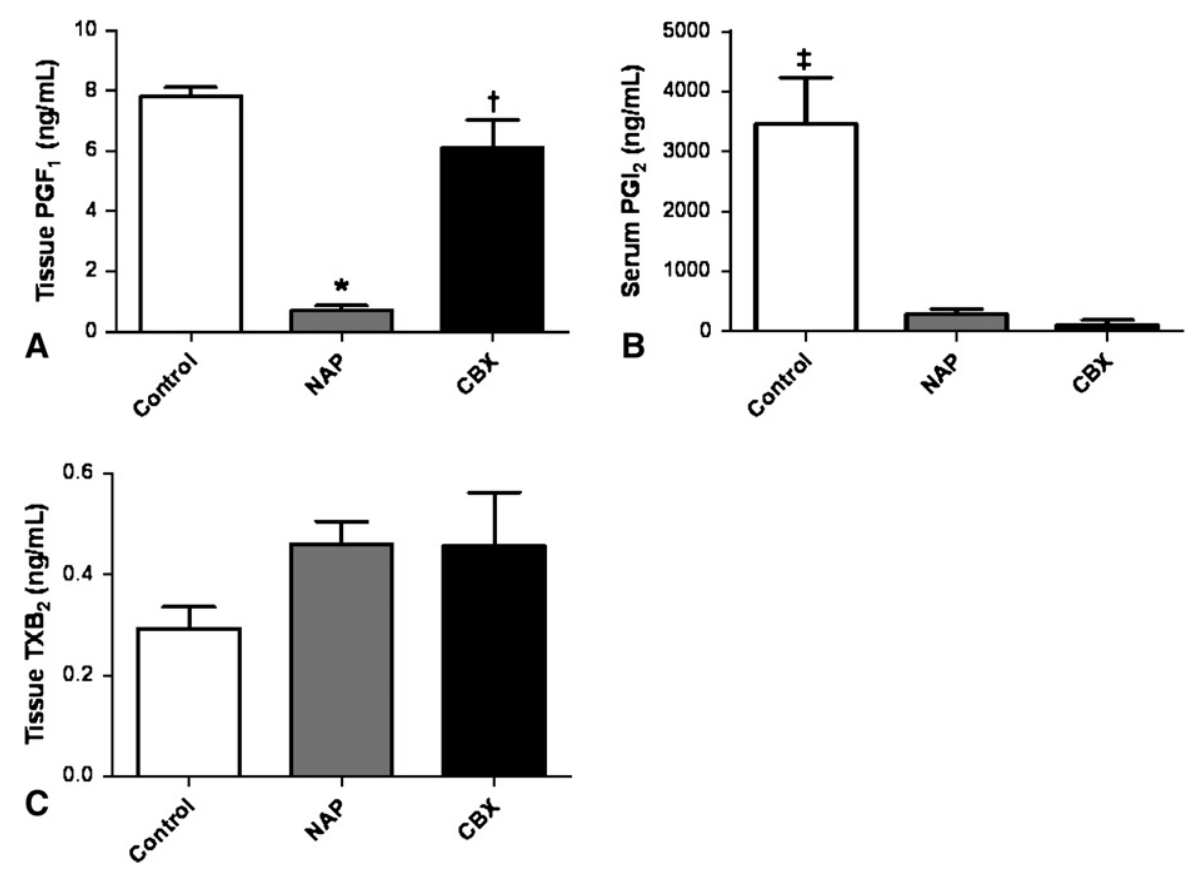

FIGURE 4. Serum and tissue levels of prostaglandins and thromboxane. A, Tissue levels of prostaglandin $\mathrm{F}_{1 \alpha}\left(P G F_{1}\right)$ show marked decrease in naproxen (NAP) group (asterisk indicates $P<.001 \mathrm{vs} \mathrm{control} \mathrm{and} \mathrm{celecoxib)} \mathrm{and} \mathrm{significant} \mathrm{decrease} \mathrm{in} \mathrm{celecoxib} \mathrm{group} \mathrm{as} \mathrm{well} \mathrm{(dagger} \mathrm{indicates} P<.05 \mathrm{vs}$ control). B, Serum prostaglandin $\left(P G I_{l a}\right)$ levels are significantly higher in control group (double dagger indicates $P=.004$ vs naproxen and celecoxib). C, Tissue levels of thromboxane $\mathrm{B}_{2}\left(T X B_{2}\right)$ are slightly increased in naproxen and celecoxib groups $(P=.25$ vs control).

\section{DISCUSSION}

The principle findings of this study are that the nonselective COX inhibitor naproxen increased collateral-dependent perfusion in a chronic model of myocardial ischemia relative to untreated animals, whereas the selective COX-2 inhibitor celecoxib did not alter collateral-dependent perfusion. Serum levels of $\mathrm{PGI}_{2}$ were reduced to a similar extent by both drugs, and tissue levels of $\mathrm{TXA}_{2}$ were slightly and similarly increased relative to untreated animals. Capillary densities in the collateral-dependent myocardium were diminished in both naproxen and celecoxib groups.

Previous studies have demonstrated that both nonselective $\mathrm{COX}^{10}$ and selective COX-2 inhibitors ${ }^{11}$ diminish coronary collateral perfusion. Most previous studies have examined the effects of indomethacin and other nonspecific COX inhibitors on acute collateral-dependent perfusion or regulation of vasomotor tone, rather than their chronic effects on collateral development. ${ }^{12}$ In our study, we treated pigs with either naproxen or celecoxib for 7 weeks. Blood flow to the collateral-dependent region was increased with nonselective COX inhibition, although there was no increase in vessel density or in the level of vasodilatory proteins. There was no difference among the groups with regard to the microvessel responses to sodium nitroprusside, an endothelium-independent vasorelaxation agent. The celecoxib group did, however, demonstrate slightly decreased relaxation responses to ADP, an endothelium-dependent vasorelaxation agent. The etiology of improved perfusion
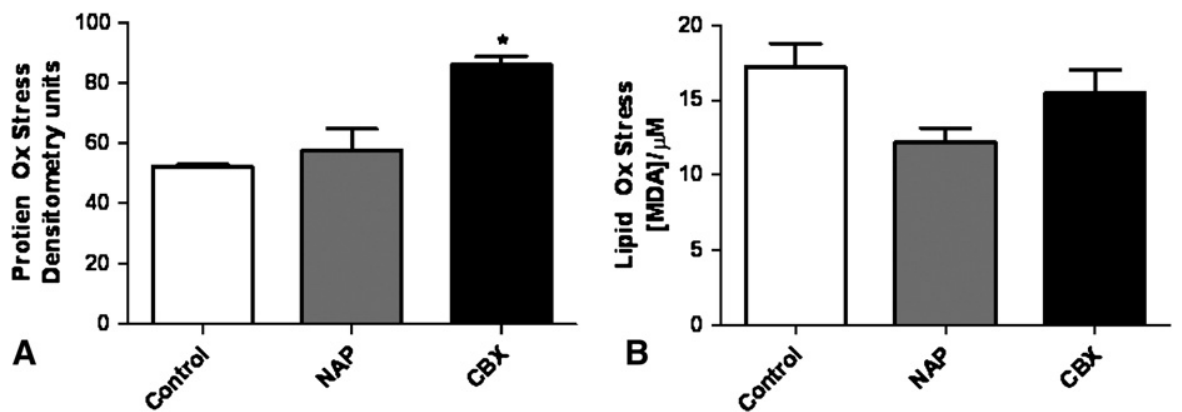

FIGURE 5. Levels of protein and lipid oxidative stress (Ox Stress) in normal and ischemic myocardium. A, Protein peroxidation levels in ischemic territory are increased in celecoxib $(C B X)$ group (asterisk indicates $P=.001$ vs control and naproxen [NAP]). B, There is trend toward decreased lipid oxidative stress in ischemic territory in naproxen group $(P=.07) . M D A$, Malondialdehyde. 
A

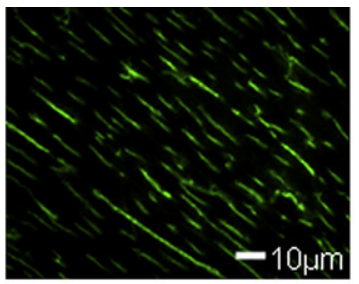

B
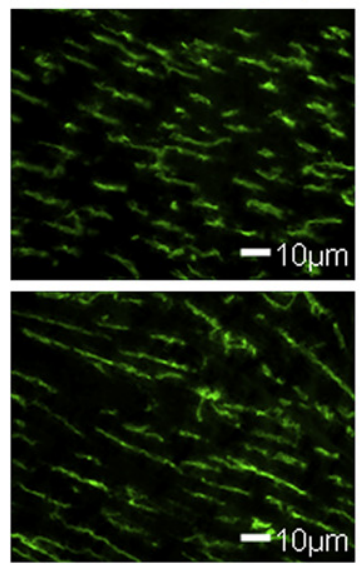
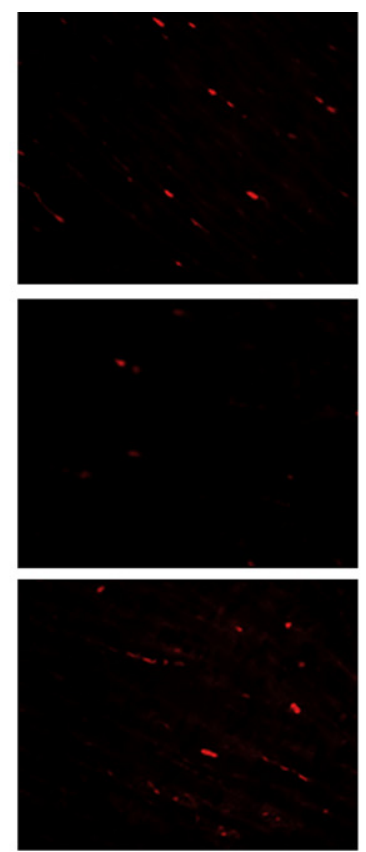
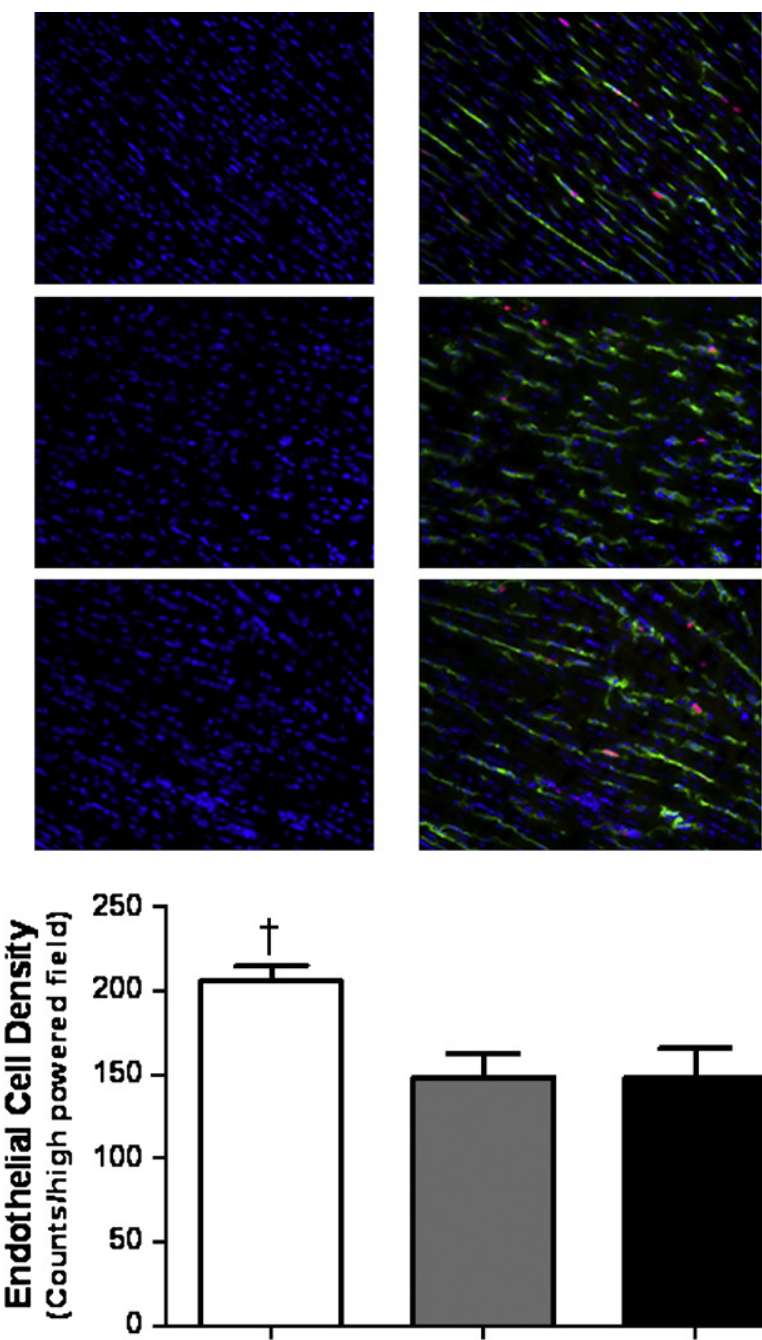

E

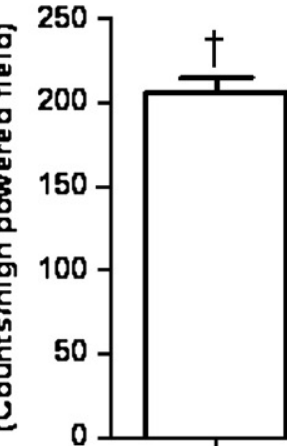

Control

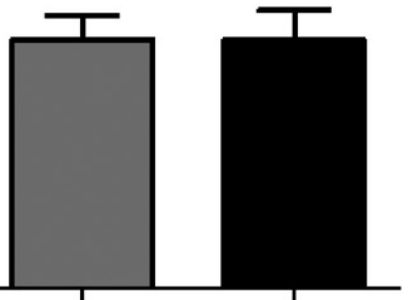

NAP

CBX

FIGURE 6. Quantification of dividing and total endothelial cells. Double staining for endothelium (CD-31, green) and dividing cells (Ki-67, red) with 4',6-diamidino-2-phenylindole (blue) allows visualization of dividing endothelial cells in ischemic territory, which appear pink in combined image. A, In this representative section for control group, there are many dividing endothelial cells. B, Representative myocardial section from ischemic area of naproxen group demonstrates fewer dividing endothelial cells. C, Representative image from celecoxib group. All images taken at $200 \times$ magnification. D, Quantification of Ki-67-positive endothelial cells demonstrates significant increases in control group relative to naproxen (NAP) and celecoxib $(C B X)$ groups $($ asterisk indicates $P=.003$ ). There is no difference between naproxen and celecoxib groups. E, Similarly, total number of endothelial cells in ischemic territory is significantly lower in naproxen and celecoxib groups than in control (dagger indicates $P=.003$ ).

to the ischemic territory in the naproxen group is not clear but may be related to preserved microvascular relaxation and withdrawal of a vasoconstrictor effect of COX inhibition. Endostatin, a powerful endogenous inhibitor of angiogenesis, has been shown to reduce blood flow locally. ${ }^{13}$ Thus it is possible that the lower expression of endostatin in the ischemic territory of the naproxen group contributed to improved blood flow.

The celecoxib group demonstrated significantly increased mean arterial and left ventricular developed pressures, as well as worsened global left ventricular function relative to the other groups. Nearly all NSAIDs are associated with the development of systemic hypertension. ${ }^{14}$ Causes of in- creased blood pressure may include sodium retention, ${ }^{15}$ decreased prostaglandin concentrations, ${ }^{16}$ and renal vasoconstriction related to local enothelin-1 production. ${ }^{17}$ In our study, naproxen was not associated with hypertension. It is unclear why animals in the celecoxib group had lower heart rates than those in the control group. We did not assess stroke volume or cardiac output, because the change in heart rate may have been compensatory for other hemodynamic changes.

The role of COX-2 as a proangiogenic factor has been well described. ${ }^{18}$ Both nonselective $\mathrm{COX}$ and selective COX-2 inhibition led to significantly decreased endothelial density and proliferation. Both drugs caused downregulation

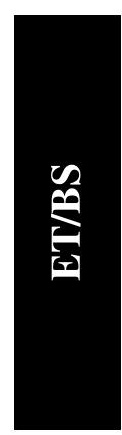



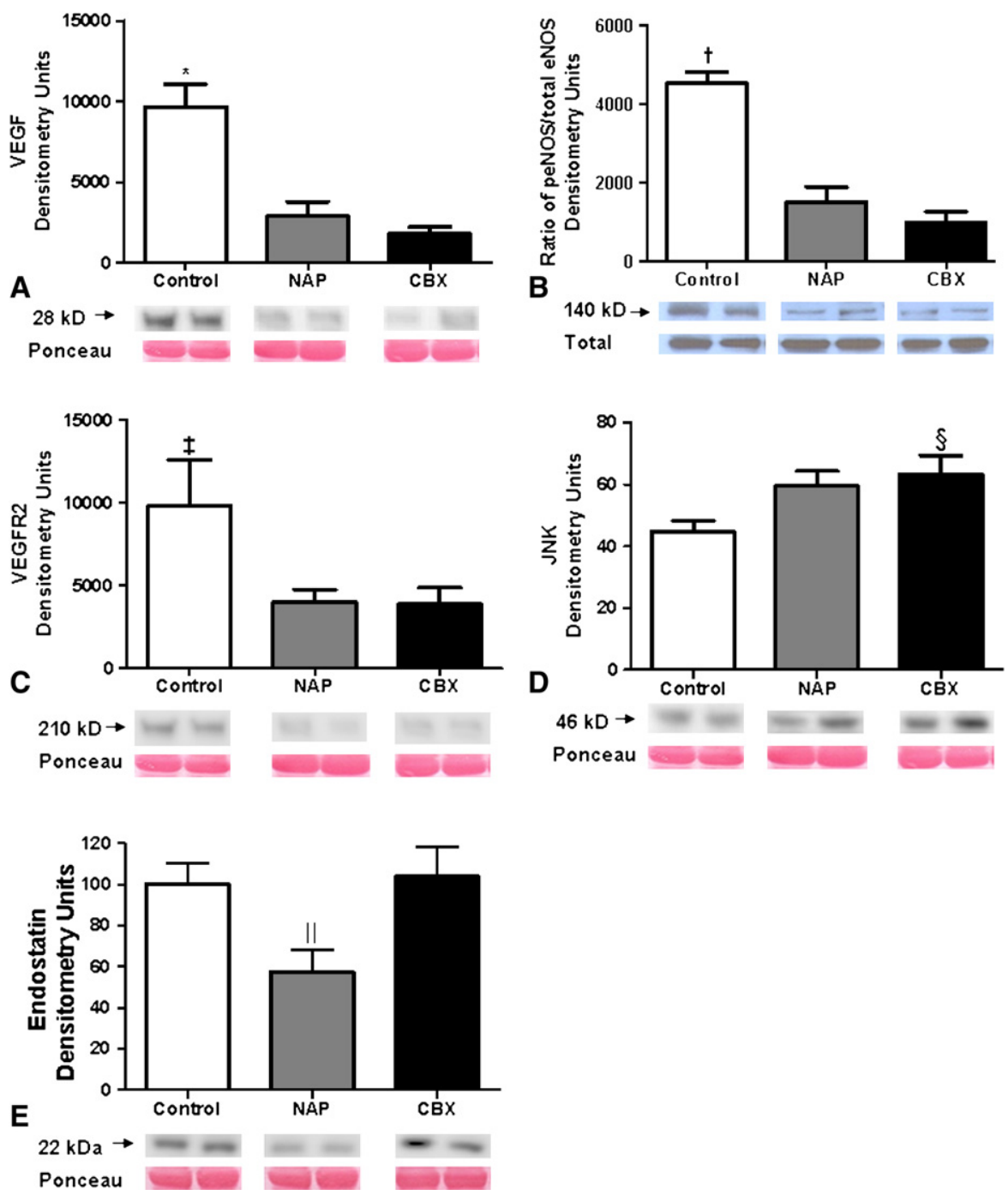

FIGURE 7. Immunoblotting in ischemic myocardium. A, Levels of vascular endothelial growth factor $(V E G F)$ are highest in control group and decreased in naproxen $(N A P)$ and celecoxib $(C B X)$ groups (asterisk indicates $P=.001$ vs naproxen and celecoxib). B, Expression of phospho-endothelial nitric oxide synthase ( $p$-eNOS) is highest in control group (dagger indicates $P=.002$ vs naproxen and celecoxib). C, Vascular endothelial growth factor receptor 2 (VEGFR2) is likewise downregulated in naproxen and celecoxib groups (double dagger indicates $P=.04$ vs naproxen and celecoxib). D, c-Jun N-terminal kinase ( $J N K$ ) expression level is increased in celecoxib group (section mark indicates $P=.04 \mathrm{vs}$ control). There is no significant difference between control and naproxen groups. E, Endostatin, antiangiogenic protein, is significantly downregulated in naproxen group relative to control and celecoxib groups (parallel lines indicate $P=.02$ ). Post hoc analysis revealed no difference between control and celecoxib groups.

of vascular endothelial growth factor, vascular endothelial growth factor receptor 2 , and phospho-endothelial nitric oxide synthase, essential components of the angiogenic pathway. The decrease in endostatin in the naproxen group was unexpected. A decrease in this protein might have resulted in increased collateral formation, but in this case it did not, at least at the capillary level. There may have also been other unmeasured antiangiogenic proteins, such as angiostatin and tumastatin, in the drug-treated groups. Surprisingly, this reduction in expression of proangiogenic mediators was not associated with a reduction in collateral-dependent perfusion. Blood flow to the myocar- dium is controlled by numerous vasoactive substances and autoregulation, which may have influenced the results.

Interestingly, we did observe an increase in expression of the proapoptotic protein JNK in the ischemic myocardium of the animals in the celecoxib group. Levels of apoptosis at the time the animals were killed were increased in the celecoxib group, and this may have been mediated in part by JNK signaling. It has been reported that JNK leads to apoptosis through Lkb1. ${ }^{19}$ JNK has been shown to be activated by increased levels of oxidative stress. ${ }^{20} \mathrm{We}$ did not see upregulation of apoptosis-inducing factor, another protein involved with apoptosis. 
Additionally, elevated levels of protein oxidative stress were found in the ischemic myocardium of animals in the celecoxib group but not the naproxen group. Previous studies have demonstrated increases in reactive oxygen species and oxidative stress with some but not all COX-2 inhibitors, and this is thought to result in higher rates of apoptosis. ${ }^{21} \mathrm{In}$ creased levels of oxidant stress have been linked to left ventricular remodeling and the development of heart failure. ${ }^{22}$ Although most NSAIDs have been implicated in increasing oxidant stress, we did not observe this in the myocardium at the dose of naproxen used in this model.

The first selective COX-2 inhibitors were introduced around 2000 . There were early concerns regarding a theoretic increased risk of cardiovascular side effects, because it was thought that $\mathrm{PGI}_{2}$ levels could be diminished while platelet and tissue levels of TX would not be altered. This hypothesis was supported by a study that demonstrated a decrease in urinary metabolites of $\mathrm{PGI}_{2}$, but no reduction in urinary metabolites of TX in healthy volunteers taking celecoxib. ${ }^{23}$ This study did not, however, consider that COX-2 produces prostaglandins such as $\mathrm{PGI}_{2}$ in many tissues, including the lungs and colon, and the concentration of urinary metabolites is not necessarily a reflection of vascular or perivascular tissue concentrations. In our study, both COX2 inhibition and nonselective COX inhibition resulted in markedly reduced serum $\mathrm{PGI}_{2}$ levels, as well as decreased $\mathrm{PGI}_{2}$ and slightly increased $\mathrm{TXA}_{2}$ levels, in the ischemic myocardium. The decreases in $\mathrm{PGI}_{2}$ tissue levels in the celecoxib group were more modest than those in the naproxen group.

The risk of adverse events may be dose dependent. For example, the Adenomatous Polyp Celecoxib trial found celecoxib to be associated with increased adverse events when administered at doses greater than those generally prescribed for arthritic conditions. ${ }^{24}$ Additionally, in a pooled analysis examining celecoxib use in about 8000 patients (approximately 16,000 patient-years of follow up) from recent randomized controlled clinical trials, the risks of cardiovascular death, myocardial infarction, stroke, heart failure, and thromboembolism were shown to be dose dependent, with hazard ratios of 1.1 for a dose of $400 \mathrm{mg}$ daily and 3.1 for a dose of $400 \mathrm{mg}$ twice a day. ${ }^{25}$ At dosages recommended for treatment of osteoarthritis (200 mg once per day), celecoxib has not been associated with an increased incidence of adverse events in any clinical trial to date. On the assumption that swine demonstrate similar dose-dependent effects, we decided to use relatively high doses of celecoxib and naproxen to create conditions similar to those in the studies in which cardiac complications were observed.

There are several limitations of this study. First, it was performed in a porcine model of chronic myocardial ischemia. Although in most situations the porcine coronary circulation closely mimics the physiology and pathophysiology of the human coronary circulation, this may not necessarily be the case for COX inhibition. Although the experimental methods in this study have been used extensively in the past, it is possible that they do not accurately represent all changes in the swine related to the drug treatment in this study. For example, the microsphere flow data may have been influenced by cardiac output. Second, a nondiseased porcine model was used, as opposed to an elderly, diabetic, or hypercholesterolemic model. Most patients with arthritic conditions requiring NSAIDs have some endothelial dysfunction related to preexisting conditions. Third, there were more animal deaths in the naproxen group than in the other groups. These animals were not included in any part of the analysis, and this may introduce a potential survival bias. Fourth, although we discontinued treatment drugs 24 hours before the final experiment, this period was not sufficient for full washout of the drug, and it is likely that residual drug was present. We attempted to minimize the acute effects of COX inhibition in an attempt to examine its longterm effects on collateral-dependent perfusion. It is likely that continuing the drugs until the time of the final experiment would have produced different findings, reflecting continued inhibition of COX or COX-2. Additionally, only a single high dose of each drug was examined, and different doses may result in different effects. Finally, not all COX-2 inhibitors display similar specificity for COX-2, and many have different toxicologic profiles. It is incorrect to assume that because a given drug in a group is associated with an adverse effect that there is a "class effect" and that all members share the effect or share it to the same degree. Drugs in this group likely function along a spectrum of potential actions on multiple pathways.

In conclusion, both a selective COX-2 and a non-selective COX inhibitor altered local and systemic physiology but did not alter myocardial perfusion relative to the control group. Although COX inhibition did not lead to reduced blood flow to the ischemic territory, both nonselective and selective inhibition led to lower levels of local vasodilators, decreased prostaglandin levels, and diminished capillary density and endothelial cell division. There were, however, some important differences between the effects of naproxen and celecoxib, such as their effects on hemodynamics, oxidative stress, apoptosis, and alteration of microvascular responses. The effects of selective and nonselective COX inhibition appear to be very complex, but on the basis of this work it appears that collateral-dependent myocardial blood flow is not reduced with COX inhibition in this model.

\section{References}

1. Coon KD, Inge LJ, Swetel K, Felton V, Stafford P, Bremner RM. Genomic characterization of the inflammatory response initiated by surgical intervention and the effect of perioperative cyclooxygenase 2 blockade. J Thorac Cardiovasc Surg. 2010;139:1253-60. 1260.e1-2.

2. Bombardier C, Laine L, Reicin A, Shapiro D, Burgos-Vargas R, Davis B, et al. Comparison of upper gastrointestinal toxicity of rofecoxib and naproxen in patients with rheumatoid arthritis. VIGOR Study Group. $N$ Engl J Med. 2000; 343:1520-8. 2 p following 1528 . 
3. Farkouh ME, Greenberg BP. An evidence-based review of the cardiovascular risks of nonsteroidal anti-inflammatory drugs. Am J Cardiol. 2009;103:1227-37.

4. Ott E, Nussmeier NA, Duke PC, Feneck RO, Alston RP, Snabes MC, et al. Efficacy and safety of the cyclooxygenase 2 inhibitors parecoxib and valdecoxib in patients undergoing coronary artery bypass surgery. $J$ Thorac Cardiovasc Surg. 2003; 125:1481-92.

5. Cheng Y, Wang M, Yu Y, Lawson J, Funk CD, Fitzgerald GA. Cyclooxygenases, microsomal prostaglandin E synthase-1, and cardiovascular function. J Clin Invest. 2006;116:1391-9.

6. Wu G, Luo J, Rana JS, Laham R, Sellke FW, Li J. Involvement of COX-2 in VEGF-induced angiogenesis via P38 and JNK pathways in vascular endothelial cells. Cardiovasc Res. 2006;69:512-9.

7. Meier P, Gloekler S, Zbinden R, Beckh S, de Marchi SF, Zbinden S, et al. Beneficial effect of recruitable collaterals: a 10-year follow-up study in patients with stable coronary artery disease undergoing quantitative collateral measurements. Circulation. 2007;116:975-83.

8. Tofukuji M, Metais C, Li J, Franklin A, Simons M, Sellke FW. Myocardial VEGF expression after cardiopulmonary bypass and cardioplegia. Circulation. 1998; 98(19 Suppl):II242-8.

9. Boodhwani M, Mieno S, Feng J, Sodha NR, Clements RT, Xu SH, Sellke FW. Atorvastatin impairs the myocardial angiogenic response to chronic ischemia in normocholesterolemic swine. J Thorac Cardiovasc Surg. 2008;135:117-22.

10. Altman JD, Klassen CL, Bache RJ. Cyclooxygenase blockade limits blood flow to collateral-dependent myocardium during exercise. Cardiovasc Res. 1995;30: 697-704.

11. Rossoni G, Muscara MN, Cirino G, Wallace JL. Inhibition of cyclo-oxygenase-2 exacerbates ischaemia-induced acute myocardial dysfunction in the rabbit. $\mathrm{Br}$ J Pharmacol. 2002;135:1540-6.

12. Dai XZ, Bache RJ. Effect of indomethacin on coronary blood flow during graded treadmill exercise in the dog. Am J Physiol. 1984;247(3 Pt 2):H452-8.

13. Sorensen DR, Read TA, Porwol T, Olsen BR, Timpl R, Sasaki T, et al. Endostatin reduces vascularization, blood flow, and growth in a rat gliosarcoma. Neuro Oncol. 2002;4:1-8.

14. Johnson AG, Nguyen TV, Day RO. Do nonsteroidal anti-inflammatory drugs affect blood pressure? A meta-analysis. Ann Intern Med. 1994;121:289-300.

15. Zhang J, Ding EL, Song Y. Adverse effects of cyclooxygenase 2 inhibitors on renal and arrhythmia events: meta-analysis of randomized trials. JAMA. 2006;296: 1619-32.

16. Funk CD, FitzGerald GA. COX-2 inhibitors and cardiovascular risk. J Cardiovasc Pharmacol. 2007;50:470-9.

17. Johnson AG, Nguyen TV, Owe-Young R, Williamson DJ, Day RO. Potential mechanisms by which nonsteroidal anti-inflammatory drugs elevate blood pressure: the role of endothelin-1. J Hum Hypertens. 1996;10:257-61.

18. Wu G, Mannam AP, Wu J, Kirbis S, Shie JL, Chen C, et al. Hypoxia induces myocyte-dependent COX-2 regulation in endothelial cells: role of VEGF. Am J Physiol Heart Circ Physiol. 2003;285:H2420-9.

19. Lee JH, Koh H, Kim M, Park J, Lee SY, Lee S, et al. JNK pathway mediates apoptotic cell death induced by tumor suppressor LKB1 in Drosophila. Cell Death Differ. 2006;13:1110-22.

20. Mendelson KG, Contois LR, Tevosian SG, Davis RJ, Paulson KE. Independent regulation of JNK/p38 mitogen-activated protein kinases by metabolic oxidative stress in the liver. Proc Natl Acad Sci U S A. 1996;93:12908-13.

21. Ryan EP, Bushnell TP, Friedman AE, Rahman I, Phipps RP. Cyclooxygenase-2 independent effects of cyclooxygenase- 2 inhibitors on oxidative stress and intracellular glutathione content in normal and malignant human B-cells. Cancer Immunol Immunother. 2008;57:347-58.

22. Suematsu N, Tsutsui H, Wen J, Kang D, Ikeuchi M, Ide T, et al. Oxidative stress mediates tumor necrosis factor- $\alpha$-induced mitochondrial DNA damage and dysfunction in cardiac myocytes. Circulation. 2003;107:1418-23.

23. McAdam BF, Catella-Lawson F, Mardini IA, Kapoor S, Lawson JA FitzGerald GA. Systemic biosynthesis of prostacyclin by cyclooxygenase (COX)-2: the human pharmacology of a selective inhibitor of COX-2. Proc Natl Acad Sci U S A. 1999;96:272-7.

24. Solomon SD, McMurray JJ, Pfeffer MA, Wittes J, Fowler R, Finn P, et al. Cardiovascular risk associated with celecoxib in a clinical trial for colorectal adenoma prevention. N Engl J Med. 2005;352:1071-80.
25. Solomon SD, Wittes J, Finn PV, Fowler R, Viner J, Bertagnolli MM, et al. Cardiovascular risk of celecoxib in 6 randomized placebo-controlled trials: the cross trial safety analysis. Circulation. 2008;117:2104-13.

\section{Discussion}

Dr Y. Joseph Woo (Philadelphia, $\mathrm{Pa}$ ). That is a very impressive amount of work. The microvascular perfusion in the NSAID group was higher than the other groups, correct?

Dr Robich. Yes, the perfusion in the myocardium, the blood flow, was increased.

Dr Woo. So how do you explain that in light of all of the other findings?

Dr Robich. One thing that we didn't look at was some of the more upstream arterials that are supplying blood flow. So we are not sure whether that is playing a part in this as well.

Dr Woo. Did you catheterize the animals?

Dr Robich. They did get catheterized, correct. There were no differences in Rentrop scores or in myocardial blush grading. So from an angiographic standpoint, there wasn't any increase in large, large vessels.

Dr Woo. So potentially something smaller than what is angiographically visible, but larger than microvascular?

Dr Robich. Exactly, exactly.

Dr John S. Ikonomidis (Charleston, SC). Can you comment on how you validated the doses that you used in this study?

Dr Robich. Well, basically the way we approached this is those dosing levels would be standard starting doses in human patients. The swine weigh about half as much, and so this would represent a higher dose of drug to the swine. Most of the clinical trials have demonstrated this increase in adverse events at higher doses of the drugs, so we wanted to use a higher dose.

Dr Ikonomidis. Have you generated a specific pharmacokinetic profile for these drugs in swine?

Dr Robich. No, we have not.

Dr Michael E. Jessen (Dallas, Tex). Did you have any animals die during the course of the experiment?

Dr Robich. We did. We had 1 die in the control group, 3 in the naproxen group, and 1 in the celecoxib group.

Dr Jessen. Did you necropsy them to find what the causes were?

Dr Robich. Yes, we did a gross necropsy of each animal, Unfortunately, a lot of times animals die in this experiment and other experiments from, we presume, a ventricular arrhythmia, because there is no real gross or histologic finding to suggest why they died. Two animals in the naproxen group and 1 in the celecoxib group showed symptoms that were similar to heart failure. They were short of breath and breathing tachypneically.

Dr Jessen. How far down the line from the placement of the constrictor did this occur?

Dr Robich. All the animals that died in this experiment died somewhere around the third or fourth week. So they were out a fair bit. Now, we analyzed that, and obviously in a very small study like this that didn't reach statistical significance. But we did have some deaths. 